\title{
Working with Lentivirus for Generation of Transgenic Bovine Embryos
}

\author{
Paulo S Monzani ${ }^{1,2 *}$, Samuel Guemra ${ }^{1,2}$, Renato Zanin ${ }^{2}$, Marcos Lima ${ }^{2}$, Paulo R Adona ${ }^{1,2}$ and Otávio M Ohashi ${ }^{2,3}$ \\ ${ }^{1}$ Center for Biological and Health Sciences, University of Northern Paraná , Londrina, Paraná , Brazil \\ ${ }^{2}$ Animal Reproduction Laboratory of Agricultural Laffranchi, Tamarana , Paraná , Brazil \\ ${ }^{3}$ Institute of Biological Sciences , Federal University of Pará, Belém, Pará, Brazil
}

Keywords: Lentivirus; Transgenic Bovine Embryos; Antibiotic Selection; Subzonal Injection; Denuded Zygotes

Pharmaceutical industry started with the production of proteins for therapeutic purposes using the extraction from producing tissues, such as insulin derived from a pig's pancreas and growth hormone from the pituitary gland. The proteins from these tissues often show low quality and increase in the risk of transmitting diseases such as HIV or those caused by prions. Recombinant DNA technology has allowed the heterologous proteins production using the bacterial system [1] that results in increased protein availability, therefore, improving quality and reducing the risk of disease transmission. Bacteria have been widely used as bioreactors because they are easily cultured. However, these microorganisms have limited ability to perform post-translational modifications of proteins necessary for the formation of biologically active molecules [2].

Mammalian cells achieve complex post-translational modifications, but the use of this system has a high cost for the large-scale protein production. Transgenic animals produce recombinant proteins with lower costs than mammalian cells. However, the establishment of production is relatively slow and still presents the same regulatory hurdles [3]. Mammary gland is considered the principal tissue for the expression of recombinant proteins and great efforts have been employed to generate transgenic animals for this purpose. Currently, two biopharmaceuticals derived from the milk of transgenic animals are commercially available: anti- $\alpha$-thrombin (ATryn) produced by GTC Biotherapeutics UK Limited and C1 esterase inhibitor (Ruconest) produced by Pharming Group NV.

Retroviruses have been used in different techniques of transgenic animal generation, such as nuclear transfer, embryonic stem cells, gene transfer mediated sperm and in the transduction of oocytes or zygotes. The size of the exogenous DNA to be inserted into the lentiviral vectors is the principal limitation of the technique, due to its packaging into a viral particle. Lentiviral transgenesis is based on the transfer of exogenous genes to embryos before implantation in the uterus. For efficient infection of mammalian zygote, the lentivirus must overcome the zona pellucidae, the extracellular matrix that surrounds the oocyte and embryo, forming a physical barrier against viral infection [4]. Two main methods to infect zygotes were reported, one consisting of subzonal injection of lentivirus in the perivitelline space [5-8], and lentivirus infection of denuded zygotes with zona pellucidae enzymatically digested $[5,9]$.

The success of these methodologies was obtained using the commercial vector FUGW. Therefore, we have adopted this vector to produce the lentiviruses and use them as a control to the standardization of zygote transduction. We have constructed other lentiviral vectors by directing the recombinant protein expression in the milk [10] and used the lentivirus to promote zygote transduction.

The lentivirus was produced in 293-FT cells, and then concentrated concentrated about 100-fold using PEG, before the transduction. The analysis by q-PCR showed that the concentration of lentivirus from
FUGW and the constructed vectors had similar quantities of lentiviral particles. The bovine embryos transduced with lentivirus from FUGW were analyzed by epifluorescence microscopy and PCR. The PCR analysis showed that the majority of the embryos were transgenic when compared with the not transduced control group. However, in the microscopy analysis, we have observed a variation from 10 to $30 \%$ of the embryos EmGFP positive (Figure 1) in the experiments. During the experiments, it was verified that analysis by epifluorescence is not completely reliable. Accordingly, bovine embryos presented natural fluorescence at the seventh day of development, and sometimes the differentiation between transgenic embryo and non-transgenic embryo is dependent on the quantity of EmGFP expression by individual embryos.

Individual embryos transduced with lentivirus from constructed vectors were analyzed by PCR (Figure 2) and nearly all embryos analyzed were positive when compared with non-transduced embryos. These results were not expected once the fibroblast transduction using

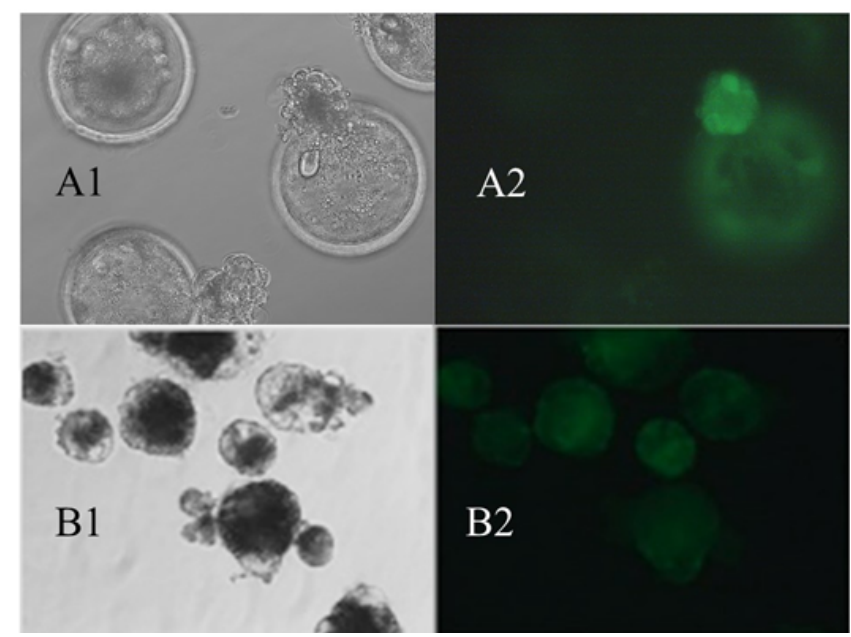

Figure 1: Analysis of EmGFP expression in blastocyst using epifluorescence microscopy. A-subzonal injection of lentivirus in the perivitelline space. A1Bright light. A2-epifluorescence. B-lentivirus infection of denuded zygotes. B1-Bright light. B2-epifluorescence.

*Corresponding author: Paul S Monzani, Center for Biological and Health Sciences, University of Northern Paraná, Londrina-PR, Brazil, Tel: 5543 33994700; E-mail: monzani.paulo@gmail.com

Received November 26, 2014; Accepted November 27, 2014; Published December 01, 2014

Citation: Monzani PS, Guemra S, Adona PR, Ohashi OM (2014) Working with Lentivirus for Generation of Transgenic Bovine Embryos. Pharm Anal Acta 5: e169. doi:10.4172/2153-2435.1000e169

Copyright: (c) 2014 Monzani PS, et al. This is an open-access article distributed under the terms of the Creative Commons Attribution License, which permits unrestricted use, distribution, and reproduction in any medium, provided the original author and source are credited. 
C1

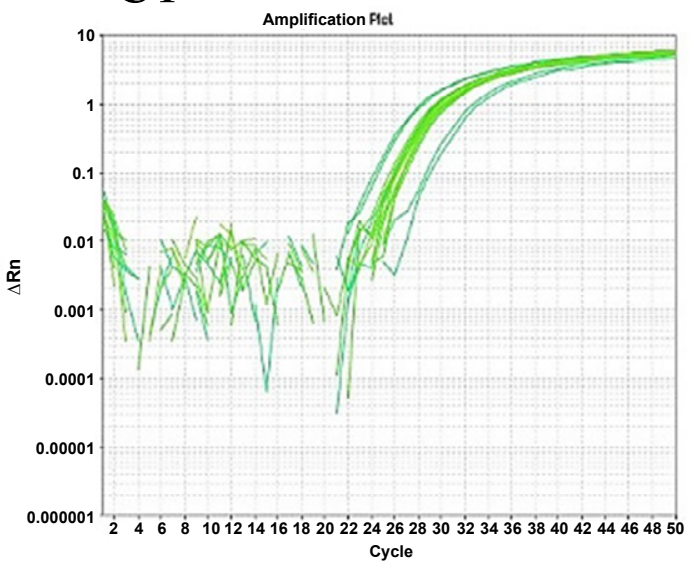

C2

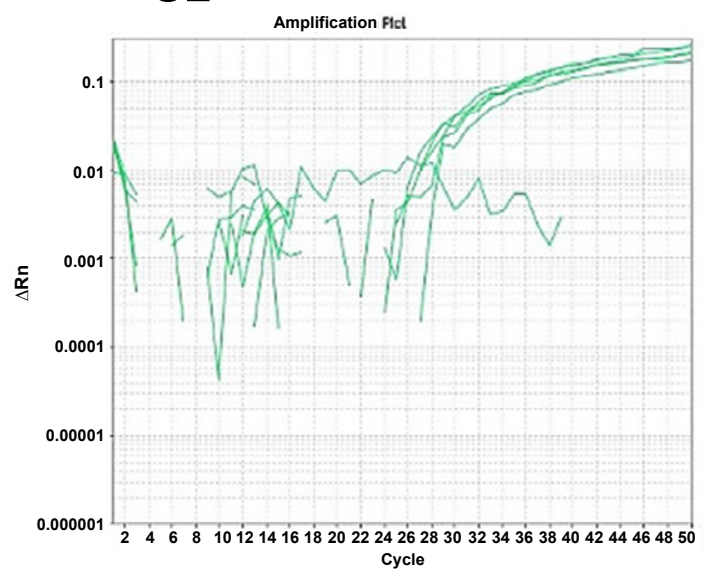

Figure 2: Analysis of individual blastocyst transgene. C1-EmGFP gene transgene. C2-Human insulin gene transgene.

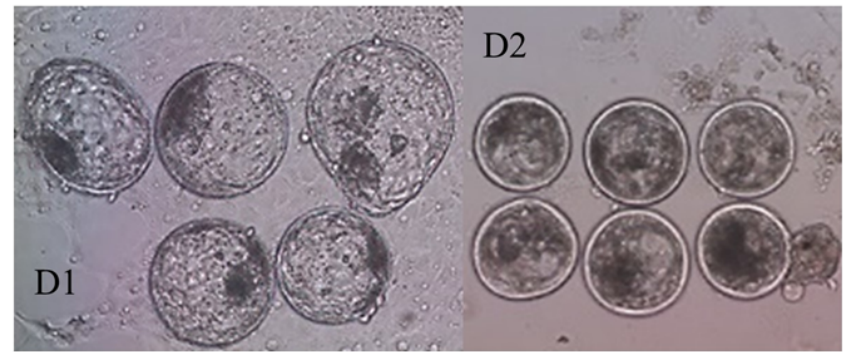

Figure 3: Kill curve of blasticidin. D1-control embryos production without blasticidin, D2-interruption of embryonic development using $6 \mu \mathrm{g} \cdot \mathrm{mL}^{-1}$ blasticidin.

these lentiviruses produced few transgenic colonies when compared with transduction using lentivirus from FUGW. We have transduced bovine zygote with zona pellucidae in cultivation with lentivirus, and again it was verified positive transgene in the majority of the embryos. After transducing experiments, we concluded that the use of PCR to evaluate single embryos was not effective to evaluate transgenic embryos. It is likely that during the lentivirus packaging, the DNA vector used in the transfection step could be packed into lentiviral particle, and that could be the cause of false positive. Intending to solve this problem, we have used the blasticidin selection to select transgenic embryos. We have created a kill curve for blasticidin (Figure 3) and it was verified that the use of $6 \mu \mathrm{g} \cdot \mathrm{mL}^{-1}$ of blasticidin, at third to seventh day of development, was enough to kill the embryos or to stop the development. The use of blasticidin has decreased the embryo production compared to the control experiment. Since the PCR analysis was not efficient to determine the transgene, we decided to transfer the embryos from blasticidin selection of the subzonal injection and lentivirus infection of denuded zygotes to the receptor cows. About ten animals were born from this procedure, and the PCR analysis showed that the animals were not transgenic.

The fact that the embryos survived to blasticidin selection could be due to the short time of selection, limited to four days while fibroblast selection it needed about ten days in the presence of blasticidin. Therefore, it is possible that the genetic material from lentivirus was introduced into the zygote's cytoplasm, which supports the fact that the embryos survived, but the integration of the transgene to the genome was not efficient. The large size, about $9 \mathrm{~kb}$, of the material to be inserted into the genome could be one of the reasons that prevented this integration. Therefore, the transgene with the size near that FUGW could improve the transduction. So, the selection using antibiotics would be effective in bovine embryos where selection could occur during a period of three days.

The lentiviral infection of denuded zygotes is a laborious methodology in addition, the embryos' production production was more variable, and the embryos' quality was worse in comparison with subzonal injection. Both techniques were established about 12 years ago, and although they are regarded as promising techniques for producing transgenic animals, so far, they have not yet been used for efficient generation of transgenic animals for the production of biopharmaceuticals in the milk.

\section{References}

1. Jensen EB, Carlsen S (1990) Production of recombinant human growth hormone in Eschericia coli: expression of different precursors and physiological effects of glucose, acetate, and salts. Biotechnol Bioeng 36: 1-11.

2. Jana S, Deb JK (2005) Strategies for efficient production of heterologous proteins in Escherichia coli. Appl Microbiol Biotechnol 67: 289-298.

3. Dyck MK, Lacroix D, Pothier F, Sirard MA (2003) Making recombinant proteins in animals-different systems, different applications. Trends Biotechnol 21: 394-399.

4. Pfeifer A (2006) Lentiviral transgenesis-A versatile tool for basic research and gene therapy. Curr Gene Ther 6: 535-542.

5. Lois C, Hong EJ, Pease S, Brown EJ, Baltimore D (2002) Germline transmission and tissue-specific expression of transgenes delivered by lentiviral vectors. Science 295: 868-872.

6. Hofmann A, Kessler B, Ewerling S, Weppert M, Vogg B, et al. (2003) Efficient transgenesis in farm animals by lentiviral vectors. EMBO Reports 4: 1054-1060.

7. Hofmann A, Zakhartchenko V, Weppert M, Sebald $H$, Wenigerkind $H$, et al (2004) Generation of Transgenic Cattle by Lentiviral Gene Transfer into Oocytes. Biol Reprod 71: 405-409.

8. Miao K, Guo M, An L, Xu XL, Wu H, et al. (2011) A new method to efficiently produce transgenic embryos and mice from low-titer lentiviral vectors. Transgenic Res 20: 357-363.

9. Pfeifer A, Ikawa M, Dayn Y, Verma IM (2002) Transgenesis by lentiviral vectors Lack of gene silencing in mammalian embryonic stem cells and preimplantation embryos. Proc Natl Acad Sci USA 99: 2140-2145.

10. Monzani PS, Guemra S, Adona PR, Ohashi OM, Meirelles FV, et al. (2015) MAC-T cells as a tool to evaluate lentiviral vector construction targeting recombinant protein expression in milk. Anim Biotechnol 26: 136-142. 